\title{
Long non-coding RNA ZEB1-AS1 predicts a poor prognosis and promotes cancer progression through the miR-200a/ZEB1 signaling pathway in intrahepatic cholangiocarcinoma
}

\author{
MINGWEN JIAO $^{1 *}$, SHANGLEI NING ${ }^{2 *}$, JINGBO CHEN $^{3 *}$, LILI CHEN $^{4}$, \\ MENG JIAO $^{5}$, ZHONGHUI CUI ${ }^{3}$, LINGYU GUO ${ }^{2}$, WENTAO MU $^{2}$ and HUI YANG ${ }^{3}$ \\ ${ }^{1}$ Department of Colorectal and Anal Surgery, Shandong Provincial Qianfoshan Hospital, \\ Shandong University; ${ }^{2}$ Department of Hepatobiliary Surgery, Qilu Hospital of Shandong University; \\ ${ }^{3}$ Department of Colorectal and Anal Surgery, The First Affiliated Hospital of Shandong First Medical University; \\ ${ }^{4}$ Department of Pathology, Jinan Central Hospital Affiliated to Shandong University, Jinan, Shandong 250012; \\ ${ }^{5}$ Department of Gastrointestinal Surgery, The Second Affiliated Hospital of Shandong First Medical University, \\ Tai'an, Shandong 271600, P.R. China
}

Received October 5, 2019; Accepted February 28, 2020

DOI: $10.3892 /$ ijo.2020.5023

\begin{abstract}
Emerging evidence suggests that long non-coding RNAs (lncRNAs) play pivotal roles in cancer progression, including in intrahepatic cholangiocarcinoma (IHCC). The overexpression of lncRNA ZEB1 antisense 1 (ZEB1-AS1) has been discovered in several types of cancer; however, the clinical significance and functional role of ZEB1-AS1 in IHCC have not yet been determined. In the present study, ZEB1-AS1 was found to be upregulated in IHCC cell lines and tissues. A high ZEB1-AS1 expression was associated with clinical progression and a poor survival of patients with IHCC, and was identified as an independent risk factor for a poor prognosis. In addition, ZEB1-AS1 promoted the proliferation and metastasis of IHCC cells both in vitro and in vivo. ZEB1-AS1 was demonstrated to increase the expression of ZEB1 by sponging miR-200a and to thereby accelerate epithelial-mesenchymal transition (EMT). On the whole, the findings of the present study demonstrate that ZEB1-AS1 promotes proliferation and metastasis in IHCC, and induces EMT through the miR-200a/ZEB1 signaling pathway. ZEB1-AS1 may thus be a promising prognostic biomarker and essential therapeutic target for IHCC.
\end{abstract}

Correspondence to: Dr Hui Yang, Department of Colorectal and Anal Surgery, The First Affiliated Hospital of Shandong First Medical University, 16766 Jingshi Road, Jinan, Shandong 250012, P.R. China

E-mail: yanghqfshospital@163.com

${ }^{*}$ Contributed equally

Key words: non-coding RNA, intrahepatic cholangiocarcinoma, progression, prognosis

\section{Introduction}

Intrahepatic cholangiocarcinoma (IHCC) is a rare and highly aggressive primary epithelial cancer arising from the bile duct within the liver (1). The incidence and mortality rates associated with IHCC have been continuously rising over the past decade (2). Patients are often diagnosed at an advanced incurable stage with lymph node metastasis and multicentric disease already present within the liver. Curative intent surgery is available for only $30-40 \%$ of patients with IHCC (1). Moreover, the 5-year survival rate is $30 \%$ even for patients who have undergone surgical resection (3) and is $<5-10 \%$ for patients with unresectable IHCC (4). The roles of adjuvant chemo-radiotherapy and targeted therapy in IHCC have not yet been well defined and these treatments may have only modest therapeutic effects $(3,5-7)$. Therefore, further investigation into the mechanisms of IHCC progression is critical in order to enable the detection of novel diagnostic biomarkers and therapeutic targets.

Long non-coding RNAs (lncRNAs) are defined as transcripts greater than 200 nucleotides in length that are not translated into proteins (8). A large number of lncRNAs have been identified in recent years. IncRNAs have been reported to play critical roles in diverse cellular processes, including transcriptional regulation in cis or trans mode, the organization of nuclear domains and the regulation of proteins or RNA molecules (9). Notably, research has suggested that IncRNAs play pivotal roles in cancer biology (10) and can act as oncogenes or tumor suppressors. Certain lncRNAs, such as MALAT1 (11), PANDA (12), H19 (13) and MEG3 (14), have been shown to be crucial regulators in a wide range of cancer types. The lncRNA ZEB1 antisense 1 (ZEB1-AS1), an antisense transcript derived from the promoter region of ZEB1, was discovered to be upregulated in hepatocellular carcinoma by Li et al in 2015 (15). Since then, ZEB1-AS1 has been demonstrated to be overexpressed in glioma (16), colorectal cancer (17), gastric cancer (18), prostate cancer (19) 
and cervical cancer (20). ZEB1-AS1 mainly functions as an oncogene and promotes cancer progression (21). ZEB1-AS1 upregulation has been shown to be associated with a poor prognosis in multiple types of cancer (22). However, to the best of our knowledge, the role of ZEB1-AS1 in IHCC has not yet been revealed.

Epithelial-mesenchymal transition (EMT) is a pivotal cellular process of epithelial cells acquiring mesenchymal features. EMT contributes to the malignant progression, invasion and metastasis of cancer cells (23). The EMT process can be influenced by various factors (24). In total, 5 miR-200 family members (miR-200s) (miR-141, miR-200a/200b/200c and miR-429) have been revealed as essential regulators of epithelial characteristics in several types of cells and tissues $(25,26)$. However, the association between miR-200s and EMT in IHCC has not yet been determined.

The present study aimed to determine the expression and clinical significance of ZEB1-AS1 in IHCC. Additionally, the present study investigated the functional roles of ZEB1-AS1 in IHCC proliferation and metastasis in vitro and in vivo. The mechanisms underlying the induction of ZEB1-AS1 in IHCC progression were identified. The findings presented herein provide novel insight into the molecular mechanisms of IHCC progression.

\section{Materials and methods}

Tissue samples. A total of 118 IHCC tissues and 20 tumor-adjacent tissues were collected at Shandong Provincial Qianfoshan Hospital, the First Hospital Affiliated with Shandong First Medical University and Qilu Hospital of Shandong University between May, 2008 and October, 2013. Tumor-adjacent tissues were obtained at a distance of least $1 \mathrm{~cm}$ from the tumor tissues, and pathological results confirmed that no cancer cells existed in the tumor-adjacent tissues. R0 resection was performed in all recruited patients. None of the patients had received any type of anticancer therapy, such as chemotherapy, radiotherapy, interventional therapy or targeted therapy. Patients diagnosed with 2 or more malignancies or who succumbed to the disease within 1 month after surgery were excluded. The final diagnosis of the patients was confirmed by pathological results obtained by 2 pathologists independently. The 8th edition of the tumor-node-metastasis (TNM) classification system of the International Union Against Cancer was utilized for clinical staging (27).

Follow-up was performed by physical examination, biochemical tests and ultrasonography at an outpatient clinic. The determination of cancer progression was achieved by computed tomography or magnetic resonance imaging. The overall survival (OS) period was defined as the time interval between the date of surgery and either the end of follow-up or the date of death. Progression-free survival (PFS) was defined as the period between the date of surgery and cancer progression. The research protocol conformed to the principles outlined in the Declaration of Helsinki. Each patient provided written and signed informed consent and the protocol of the study was approved by the Ethics Committee of Shandong Provincial Qianfoshan Hospital, the First Hospital Affiliated with Shandong First Medical University.
Cells, cell culture and treatment. Human IHCC cell lines (HuH28, HuCCT1, RBE, CCLP-1 and HCCC-9810) and a normal human intrahepatic bile duct epithelial cell line (HIBEC) were purchased from the Cell Bank of Chinese Academy of Sciences, Shanghai Branch. The 293 Phoenix-Ampho packaging cell line was purchased from the American Type Culture Collection (ATCC). Cells were cultured in RPMI-1640 medium (Invitrogen; Thermo Fisher Scientific, Inc.) (RBE and HCCC-9810) or Dulbecco's modified Eagle's medium (DMEM, Invitrogen; Thermo Fisher Scientific, Inc.) (HuH28, HuCCT1, CCLP-1, HIBEC and 293 Phoenix-Ampho packaging cells) supplemented with $10 \%$ fetal bovine serum (FBS) (Gibco, Thermo Fisher Scientific, Inc.). The cells were cultured in $5 \% \mathrm{CO}_{2}$ and $90 \%$ humidity at $37^{\circ} \mathrm{C}$.

Cell transfection. The full-length cDNA of ZEB1-AS1 and two short hairpin RNAs (shRNAs) against human ZEB1-AS1 were synthesized by GenePharma Co. Ltd. pBabe.puro retroviral vectors containing ZEB1-AS1 cDNA or shRNA against human ZEB1-AS1 were constructed as previously described (28). The shRNA sequences were as follows: shZEB1-AS1-1, 5'-AAG UUCAAUCUCAUUGAAGUC-3' (antisense), 5'-CUUCAA UGAGAUUGAACUUCA-3' (sense); shZEB1-AS1-2, 5'-AAC UUCUAGCCUCUCUUUCAA-3' (antisense), 5'-GAAAGA GAGGCUAGAAGUUCC-3' (sense). The RBE cells in which ZEB1-AS1 was stably overexpressed and the HuCCT1 cells in which ZEB1-AS1 was stably knocked down were generated using retroviral vectors. Retrovirus was produced by transiently transfecting the 293 Phoenix-Ampho packaging cells using Lipofectamine ${ }^{\circledR} 2000$ (Thermo Fisher Scientific, Inc.) according to the manufacturer's protocol. Briefly, the virus-containing the supernatant was pooled, filtered and added to RBE or HuCCT1 cells; infected cells were then treated with puromycin $(2 \mu \mathrm{g} / \mathrm{ml})$ for 2 days and surviving cells were maintained in complete medium with puromycin $(0.5 \mu \mathrm{g} / \mathrm{ml})$.

miR-200a mimics, miR-200a inhibitors and negative controls (mimics NC and inhibitor NC) were purchased from GenePharma Co. Ltd. When cell confluence reached $50 \%$, oligonucleotide transfection was performed using Lipofectamine ${ }^{\circledR} 2000$ (Thermo Fisher Scientific, Inc.) according to the manufacturer's protocol. Cells were subjected to subsequent experimentation at $48 \mathrm{~h}$ following transfection.

Western blot analysis. Total protein was extracted from the cells using radioimmunoprecipitation assay lysis buffer (Sigma-Aldrich, Merck KGaA) containing protease inhibitors (Roche Diagnostics). An equal amount of total protein $(20 \mu \mathrm{g})$ was loaded per lane and samples were separated by $10 \%$ SDS-PAGE and then transferred to a polyvinylidene difluoride membrane (Roche Diagnostics). After being blocked with 5\% skimmed milk for $1 \mathrm{~h}$ at room temperature, the membranes were probed with primary antibodies against ZEB1 (1:3,000, cat. no. ab180905, Abcam), E-cadherin (1:1,000, cat. no. 24E10, Cell Signaling Technology, Inc.), vimentin (1:1,000, cat. no. 3932, Cell Signaling Technology, Inc.), fibronectin (1:1,000, cat. no. ab2413, Abcam), $\alpha$-catenin (1:1,000, cat. no. 2131, Cell Signaling Technology, Inc.), N-cadherin (1:1,000, cat. no. 13116, Cell Signaling Technology, Inc.) or $\beta$-actin $(1: 2,000$, 
Table I. Association between the expression level of ZEB1-AS1 and clinicopathological features of patients with IHCC.

ZEB1-AS1 expression

\begin{tabular}{|c|c|c|c|c|}
\hline Parameters & No. of patients $(n=118)$ & $\operatorname{High}(n=64)$ & Low $(n=54)$ & $\mathrm{P}$-value \\
\hline Age & & & & 0.611 \\
\hline$<55$ years & 56 & 29 & 27 & \\
\hline$\geq 55$ years & 62 & 35 & 27 & \\
\hline Sex & & & & 0.112 \\
\hline Male & 64 & 39 & 25 & \\
\hline Female & 54 & 25 & 29 & \\
\hline CA19-9 & & & & 0.479 \\
\hline$<37 \mathrm{U} / \mathrm{ml}$ & 57 & 29 & 28 & \\
\hline$\geq 37 \mathrm{U} / \mathrm{ml}$ & 61 & 35 & 26 & \\
\hline No. of tumors & & & & 0.239 \\
\hline Single & 95 & 49 & 46 & \\
\hline Multiple & 23 & 15 & 8 & \\
\hline Microvascular invasion & & & & 0.020 \\
\hline No & 65 & 29 & 36 & \\
\hline Yes & 53 & 35 & 18 & \\
\hline Encapsulation & & & & 0.612 \\
\hline Absent & 43 & 22 & 21 & \\
\hline Present & 75 & 42 & 33 & \\
\hline Differentiation grade & & & & 0.135 \\
\hline Well & 46 & 21 & 25 & \\
\hline Moderate/poor & 72 & 43 & 29 & \\
\hline Tumor size & & & & 0.201 \\
\hline$<5 \mathrm{~cm}$ & 58 & 28 & 30 & \\
\hline$\geq 5 \mathrm{~cm}$ & 60 & 36 & 24 & \\
\hline Lymphatic metastasis & & & & 0.036 \\
\hline Yes & 33 & 23 & 10 & \\
\hline No & 85 & 41 & 44 & \\
\hline TNM stage & & & & 0.037 \\
\hline $\mathrm{I} / \mathrm{II}$ & 62 & 28 & 34 & \\
\hline $\mathrm{III} / \mathrm{V}$ & 56 & 36 & 20 & \\
\hline
\end{tabular}

ZEB1-AS1, lncRNA ZEB1 antisense 1; IHCC, intrahepatic cholangiocarcinoma.

cat. no. 8457, Cell Signaling Technology, Inc.) overnight at $4^{\circ} \mathrm{C}$. Subsequently, the membranes were incubated with anti-mouse (1:3,000, cat. no. 7076, Cell Signaling Technology, Inc.) or anti-rabbit (1:3,000, cat. no. 7074, Cell Signaling Technology, Inc.) horseradish peroxidase-conjugated secondary antibodies at $37^{\circ} \mathrm{C}$ for $1 \mathrm{~h}$. Finally, the immunoreactive bands were visualized using the ECL Western blot substrate (Promega Corpo.) and a FluorChem E system (Protein Simple).

Reverse transcription-quantitative PCR (RT-qPCR). TRIzol reagent (Thermo Fisher Scientific, Inc.) was used to extract total RNA from the cells and tissues according to the manufacturer's protocol. RNA purity was evaluated based on the A260/A280 ratio. RNA was reverse transcribed into cDNA using a TaqMan ${ }^{\circledR}$ MicroRNA reverse transcription kit (Thermo
Fisher Scientific, Inc.) according to the manufacturer's instructions. The relative expression levels of the target genes were determined by qPCR using SYBR Premix Ex Taq (Takara Biotechnology Co., Ltd.). The thermocycling conditions were as follows: Initial denaturation at $95^{\circ} \mathrm{C}$ for $30 \mathrm{sec}$; 40 cycles of $5 \mathrm{sec}$ at $95^{\circ} \mathrm{C} ; 1 \mathrm{~min}$ at $60^{\circ} \mathrm{C}$ and $72^{\circ} \mathrm{C}$ for $15 \mathrm{sec}$; with a final extension cycle at $72^{\circ} \mathrm{C}$ for $5 \mathrm{~min}$. The relative levels were calculated using the $2^{-\Delta \Delta \mathrm{Cq}}$ method (29). The endogenous control gene was glyceraldehyde-3-phosphate dehydrogenase (GAPDH). The primer sequences used are listed in Table SI.

Immunohistochemistry (IHC). IHC was conducted as described in a previous study (27). Briefly, the sections were fixed with $10 \%$ formalin for $24 \mathrm{~h}$ at room temperature. The paraffin-embedded sections ( $5 \mu \mathrm{m}$ in thickness) were 
Table II. Univariate analysis of the risk factors for poor overall survival and progression-free survival of patients with IHCC.

\begin{tabular}{|c|c|c|c|c|}
\hline \multirow[b]{2}{*}{ Parameters } & \multicolumn{2}{|c|}{ Overall survival } & \multicolumn{2}{|c|}{ Progression-free survival } \\
\hline & $\operatorname{HR}(95 \% \mathrm{CI})$ & P-value & $\operatorname{HR}(95 \% \mathrm{CI})$ & P-value \\
\hline Age, $\geq 55$ vs. $<55$ years & $1.000(0.664-1.506)$ & 0.999 & $0.989(0.660-1.482)$ & 0.956 \\
\hline Sex, female vs. male & $0.928(0.614-1.402)$ & 0.722 & $0.948(0.630-1.425)$ & 0.797 \\
\hline CA19-9, $\geq 37 \mathrm{U} / \mathrm{ml}$ vs. $<37 \mathrm{U} / \mathrm{ml}$ & $1.069(0.709-1.612)$ & 0.749 & $1.090(0.726-1.637)$ & 0.676 \\
\hline No. of tumors, multiple vs. single & $2.091(1.267-3.451)$ & 0.004 & $1.945(1.181-3.203)$ & 0.009 \\
\hline Microvascular invasion, yes vs. no & $2.015(1.327-3.060)$ & 0.001 & $1.998(1.320-3.024)$ & 0.001 \\
\hline Encapsulation, present vs. absent & $1.194(0.785-1.816)$ & 0.407 & $1.217(0.804-1.842)$ & 0.353 \\
\hline Differentiation, poor/moderate vs. well & $1.681(1.081-2.613)$ & 0.021 & $1.596(1.035-2.462)$ & 0.034 \\
\hline Tumor size, $\geq 5$ vs. $<5 \mathrm{~cm}$ & $1.384(0.918-2.087)$ & 0.121 & $1.439(0.958-2.163)$ & 0.079 \\
\hline Lymphatic metastasis, yes vs. no & $3.130(2.006-4.886)$ & $<0.001$ & $3.297(2.114-5.142)$ & $<0.001$ \\
\hline TNM stage, III/IV vs. I/II & $2.662(1.749-4.053)$ & $<0.001$ & $2.616(1.725-3.969)$ & $<0.001$ \\
\hline ZEB1-AS1 expression, high vs. low & $1.211(1.114-1.316)$ & $<0.001$ & $1.185(1.093-1.285)$ & $<0.001$ \\
\hline
\end{tabular}

ZEB1-AS1, lncRNA ZEB1 antisense 1; IHCC, intrahepatic cholangiocarcinoma.

incubated with primary antibodies against ZEB1 (1:150, cat. no. ab180905, Abcam) or E-cadherin (1:400, cat. no. 24E10, Cell Signaling Technology, Inc.) overnight at $4^{\circ} \mathrm{C}$. The samples were then treated with a biotinylated secondary antibody (cat. no. SAP-9100, ZSGB-BIO) for $40 \mathrm{~min}$ at $37^{\circ} \mathrm{C}$ according to the manufacturer's protocol. The reactions were developed using a SignalStain ${ }^{\circledR}$ DAB substrate kit (cat. no. 8059, Cell Signaling Technology, Inc.). Immunostained sections were lightly counterstained with hematoxylin for $2 \mathrm{~min}$ at room temperature, dehydrated in ethanol and cleared in xylene. The scoring of immunostaining was conducted based on the staining intensity and the percentage of positively stained area. The percentage of positively stained area was scored as follows: 0 , no IHC signal at all; $1,<10 \%$ of cells stained; $2,10-50 \%$ of cells stained; and $3,>50 \%$ of cells stained. IHC intensity was scored as follows: 0 , no IHC signal; 1 , weak IHC signal; 2, moderate IHC signal; and 3, strong IHC signal. Five randomly selected high-power fields (x400 magnification) were photographed for each IHC slide using a light microscope (Olympus Corp.). The overall quantification of the IHC score was obtained by multiplying the average intensity and percentage scores of 5 different high-power fields with the maximum possible score being 9 .

Cell proliferation assays. The cell proliferative ability was evaluated using the Cell Counting kit (CCK-8) assay and the colony formation assay. For the CCK- 8 assay, cells (3,000 per well) were seeded in 96-well plates and incubated at different time points $(0,24,48,72$ and $96 \mathrm{~h})$., CCK-8 reagent $(10 \mu \mathrm{l})$ was added to each well. Subsequently, the cells were incubated for an additional $4 \mathrm{~h}$ at $37^{\circ} \mathrm{C}$. The absorbance was determined at $450 \mathrm{~nm}$ using a microplate spectrophotometer (Thermo Fisher Scientific, Inc.).

For the colony formation assay, the cells were seeded in 6-well plates $\left(1,000\right.$ cells per well) and incubated at $37^{\circ} \mathrm{C}$ for 7 days. Neutral formalin $(10 \%)$ was then used to fix the cells and crystal violet (cat. no. C8470, Solarbio) was used for staining (for $30 \mathrm{~min}$ ) at room temperature. Finally, the cells were photographed under a microscope (Olympus Corp.).

Wound healing assay. Cells were seeded in 6-well plates and cultured until reaching $80 \%$ confluence. To create the wound, the layer of cells was scratched with a sterile $20 \mu \mathrm{l}$ pipette tip. The cells were then incubated in fresh medium containing $0.5 \%$ FBS for $48 \mathrm{~h}$. The scratch was imaged at 0 and $48 \mathrm{~h}$ under a fluorescence microscope (Olympus Corp.).

Cell migration and invasion assays. For the Transwell migration assay, cells $\left(1 \times 10^{5}\right)$ in $200 \mu$ of serum-free medium were seeded in the top compartment of a Boyden chamber $(8-\mu \mathrm{m}$ pore size; Corning Inc.). The lower compartment was filled with $700 \mu \mathrm{l}$ of medium supplemented with 10\% FBS (Gibco; Thermo Fisher Scientific, Inc.) as a chemoattractant. Following $24 \mathrm{~h}$ of incubation at $37^{\circ} \mathrm{C}$, cells remaining on the upper side of the membrane were removed with a cotton swab, and the cells that had migrated to the lower side of the membrane were fixed with $70 \%$ ethanol for $20 \mathrm{~min}$ and stained with $0.1 \%$ crystal violet for $20 \mathrm{~min}$ at room temperature. The cells were then counted and imaged using a light microscope (Olympus Corp.). The protocol of the invasion assay was similar to that of the migration assay except that in the invasion assay, the Boyden chamber was precoated with Matrigel (BD Biosciences) before seeding the cells.

Luciferase reporter assay. Potential ZEB1-AS1-miRNA interactions were predicted with starBase2.0 software. The QuikChange Site-directed Mutagenesis kit (Stratagene, Agilent Technologies, Inc.) was used to generate mutations in potential miR-200a-binding sites. The potential binding sequence of miR-200a in the ZEB1-AS1 gene and the mutant sequence were amplified by PCR and cloned into the pmirGLO dual-luciferase vector (Promega Corp.); the constructs were named ZEB1-AS1-WT and ZEB1-AS1-Mut, respectively. For co-transfection, 293 cells at a confluence of $60-80 \%$ were 
Table III. Multivariate analysis of the independent risk factors for poor overall survival and progression-free survivalof patients with IHCC.

Overall survival

Parameters

No. of tumors, multiple vs. single

Microvascular invasion, yes vs. no

Differentiation, poor/moderate vs. well

Lymphatic metastasis, yes vs. no

TNM stage, III/IV vs. I/II

ZEB1-AS1 expression, high vs. low

Overall survival

HR $(95 \% \mathrm{CI}) \quad$ P-value

$\begin{array}{ll}1.837(1.090-3.095) & 0.022 \\ 1.095(0.678-1.769) & 0.710 \\ 0.977(0.611-1.626) & 0.991 \\ 1.547(0.863-2.771) & 0.143 \\ 1.877(1.099-3.205) & 0.021 \\ 1.157(1.059-1.265) & 0.001\end{array}$

B
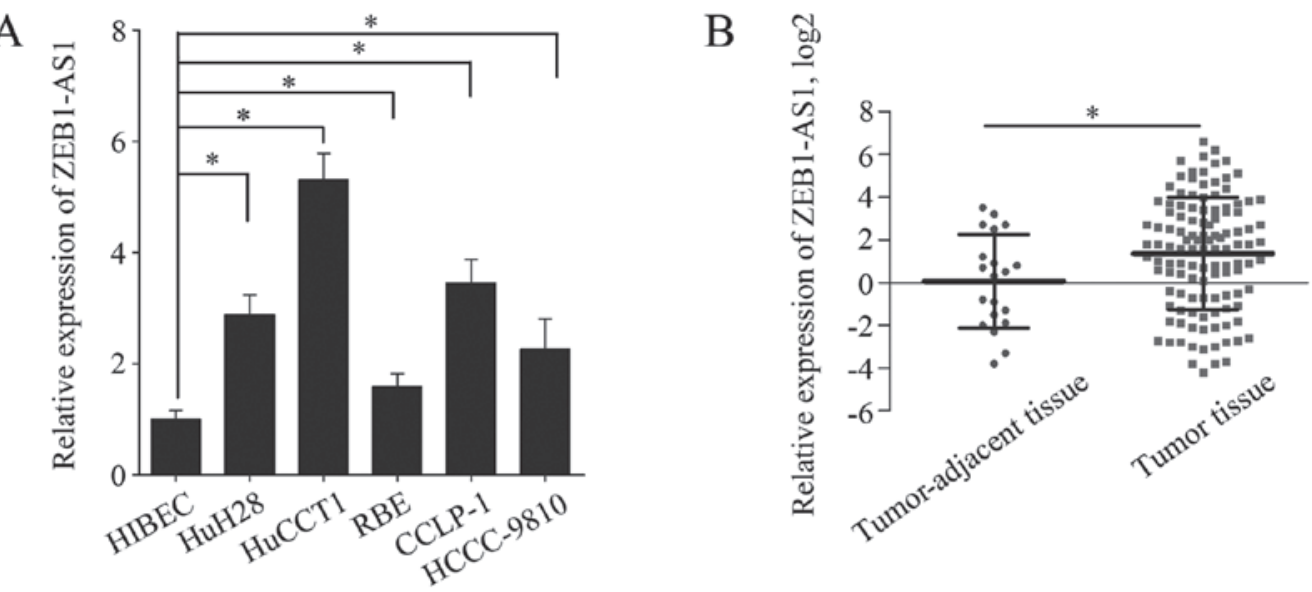

C

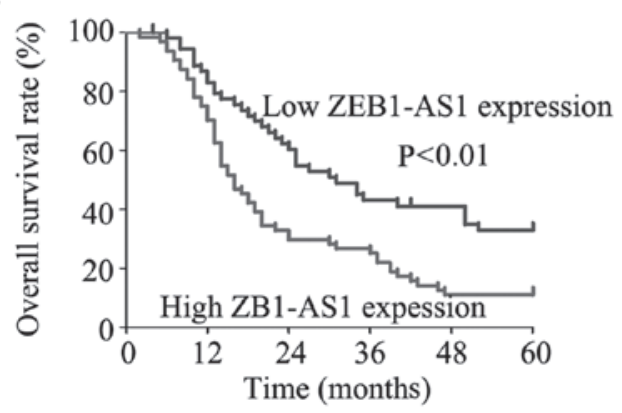

$\mathrm{D}$

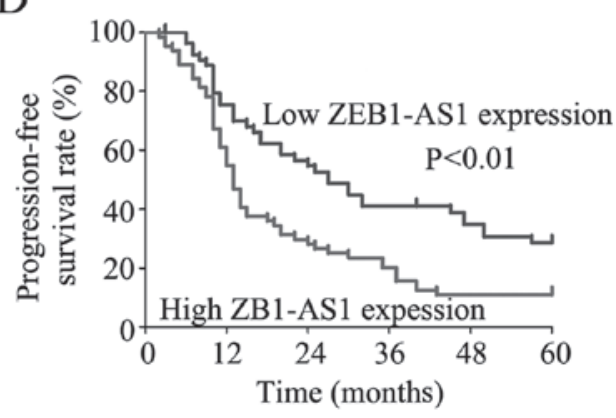

Progression-free survival

\begin{tabular}{cc}
\hline HR $(95 \% \mathrm{CI})$ & P-value \\
$1.556(0.927-2.613)$ & 0.094 \\
$1.119(0.694-1.806)$ & 0.644 \\
$0.988(0.614-1.588)$ & 0.959 \\
$1.715(0.980-3.127)$ & 0.058 \\
$1.777(1.049-3.010)$ & 0.032 \\
$1.131(1.038-1.232)$ & 0.005 \\
\hline
\end{tabular}

Figure 1. ZEB1-AS1 is upregulated in IHCC and a high ZEB1-AS1 expression predicts a poor prognosis of patients with IHCC. (A) Relative expression of ZEB1-AS1 was detected in HIBECs and 5 IHCC cell lines by RT-qPCR. (B) Relative expression of ZEB1-AS1 was measured in tumor-adjacent tissues $(n=20)$ and tumor tissues $(n=118)$ by RT-qPCR. (C) OS of patients with IHCC with a low and high ZEB1-AS1 expression. (D) PFS of patients with IHCC with a low and high ZEB1-AS1 expression. "P<0.05. ZEB1-AS1, lncRNA ZEB1 antisense 1; IHCC, intrahepatic cholangiocarcinoma; OS, overall survival; PFS, progression-free survival.

transfected with ZEB1-AS1-WT, ZEB1-AS1-Mut (50 ng) and miR-200a mimics (150 ng) using Lipofectamine ${ }^{\circledR} 2000$ (Invitrogen; Thermo Fisher Scientific). Luciferase assays were conducted at $48 \mathrm{~h}$ following transfection using the Dual Luciferase Reporter Assay System (Promega Corp.) and Renilla luciferase activity was normalized to Firefly luciferase activity.

In vivo assay. Male nude mice (BALB/c nu/nu; aged 5-6 weeks; weight, 18-22 g; 6 per group) were purchased from Shanghai Experimental Animal Center (Shanghai,
China) and housed under a 12-h light/12-h dark cycle and sterile conditions (temperature, $26-28^{\circ} \mathrm{C}$; humidity, 40-60\%) with ad libitum access to water and food. For the tumor proliferation assay, xenograft tumors were generated via the subcutaneous injection of $3.0 \times 10^{6}$ cells into the hind limbs of the nude mice. Tumor growth was determined with a caliper every 7 days. After 42 days, the mice were sacrificed and images of the tumors were captured. Tumor volume (V) was calculated as follows: $\mathrm{V}=$ largest diameter $\mathrm{x}$ (smallest diameter $)^{2} \times 0.5$. For the tumor metastasis assay, suspensions of the cells $\left(3.0 \times 10^{6}\right)$ in phosphate-buffered saline were 
A

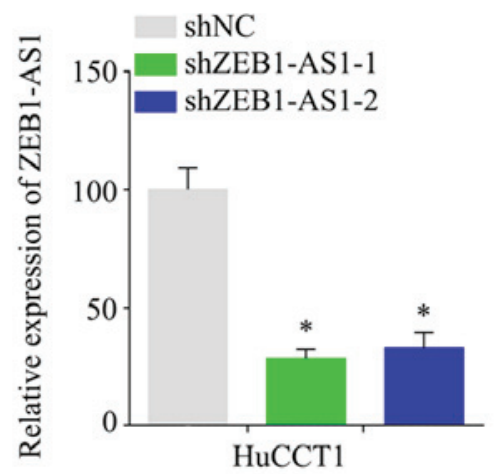

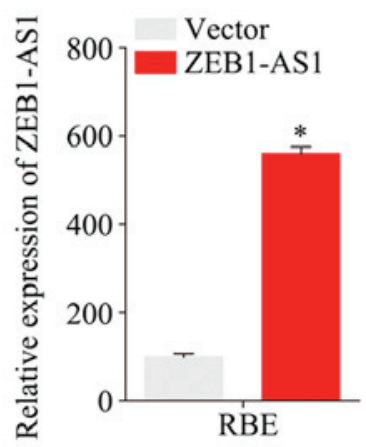

B

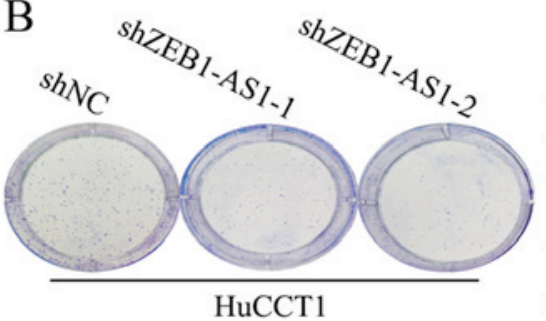

D

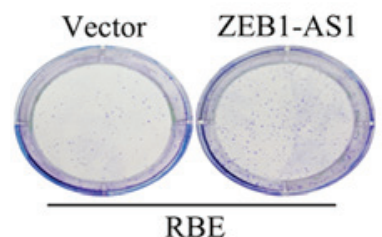

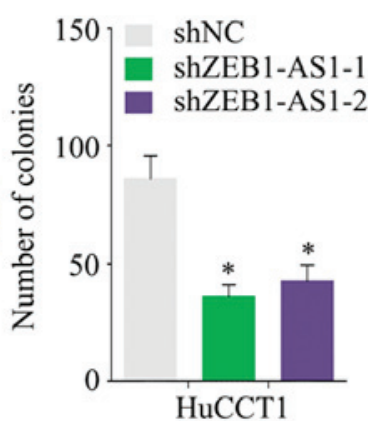

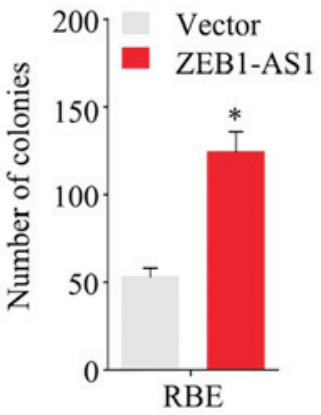

C

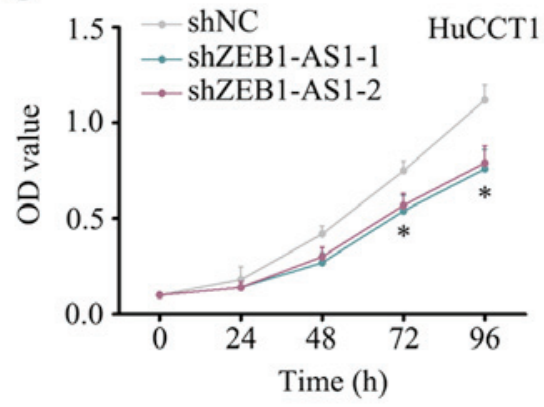

E

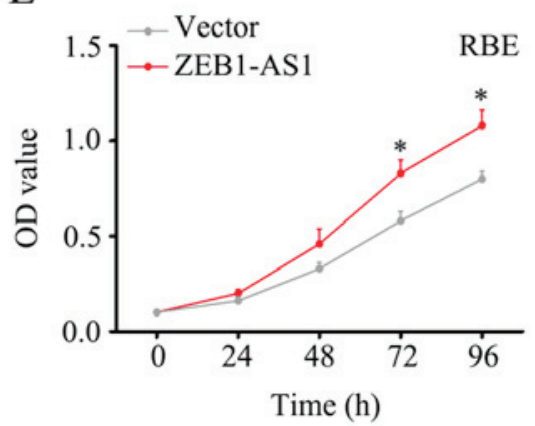

Figure 2. ZEB1-AS1 promotes the proliferation of IHCC cells. (A) Relative expression levels of ZEB1-AS1 in HuCCT1 cells with ZEB1-AS1 knockdown and RBE cells with ZEB1-AS1 overexpression. (B) Colonies of HuCCT1 cells with ZEB1-AS1 knockdown and control cells in the colony formation assay. (C) OD value of HuCCT1 cells with ZEB1-AS1 knockdown and control cells in the CCK-8 assay. (D) Colonies of RBE cells with ZEB1-AS1 overexpression and control cells in the colony formation assay. (E) OD value of RBE cells with ZEB1-AS1 overexpression and control cells in the CCK-8 assay. ${ }^{*}<0.05$. ZEB1-AS1, lncRNA ZEB1 antisense 1; IHCC, intrahepatic cholangiocarcinoma; OD, optical density.

injected into the tail veins of host mice. After 6 weeks, the animals were sacrificed, the lungs and livers were dissected out, and metastasis was evaluated. The animal experiment was approved by the Ethics Committee of Shandong Provincial Qianfoshan Hospital.

Statistical analysis. Summarized data are presented as the means \pm standard error of the mean (SEM). Differences between groups were evaluated using the $\chi^{2}$ test, Student's t-test, or one-way analysis of variance with the Least-Significant Difference correction. Linear regressions were evaluated using Spearman rank correlation analysis. Survival curves were generated using the Kaplan-Meier method and differences between these curves were evaluated with the log-rank test. Univariate and multivariate Cox proportional hazard regression models were conducted to identify independent prognostic factors. In all cases, $\mathrm{P}<0.05$ was considered to indicate a statistically significant difference. All statistical analyses were performed using GraphPad Prism 5.02 (GraphPad Software, Inc.) or SPSS 16.0 software (SPSS, Inc.).

\section{Results}

Upregulation of ZEB1-AS1 is associated with cancer progression and predicts a poor prognosis of patients with IHCC. The expression of ZEB1-AS1 was measured in the HIBECs and in 5 IHCC cell lines by RT-qPCR. The level of ZEB1-AS1 was higher in the 5 IHCC cell lines than in the HIBECs (Fig. 1A). Moreover, ZEB1-AS1 was overexpressed in the IHCC tissues $(n=118)$ relative to the tumor-adjacent tissues $(n=20)$ (Fig. 1B). To investigate the role of ZEB1-AS1 in IHCC, the patients from whom the IHCC samples were collected were divided into a high ZEB1-AS1 expression group ( $\mathrm{n}=64)$ and a low ZEB1-AS1 expression group $(n=54)$, with the mean ZEB1-AS1 expression level serving as the cut-off value. Of note, a high ZEB1-AS1 expression was identified to be associated with microvascular 
A
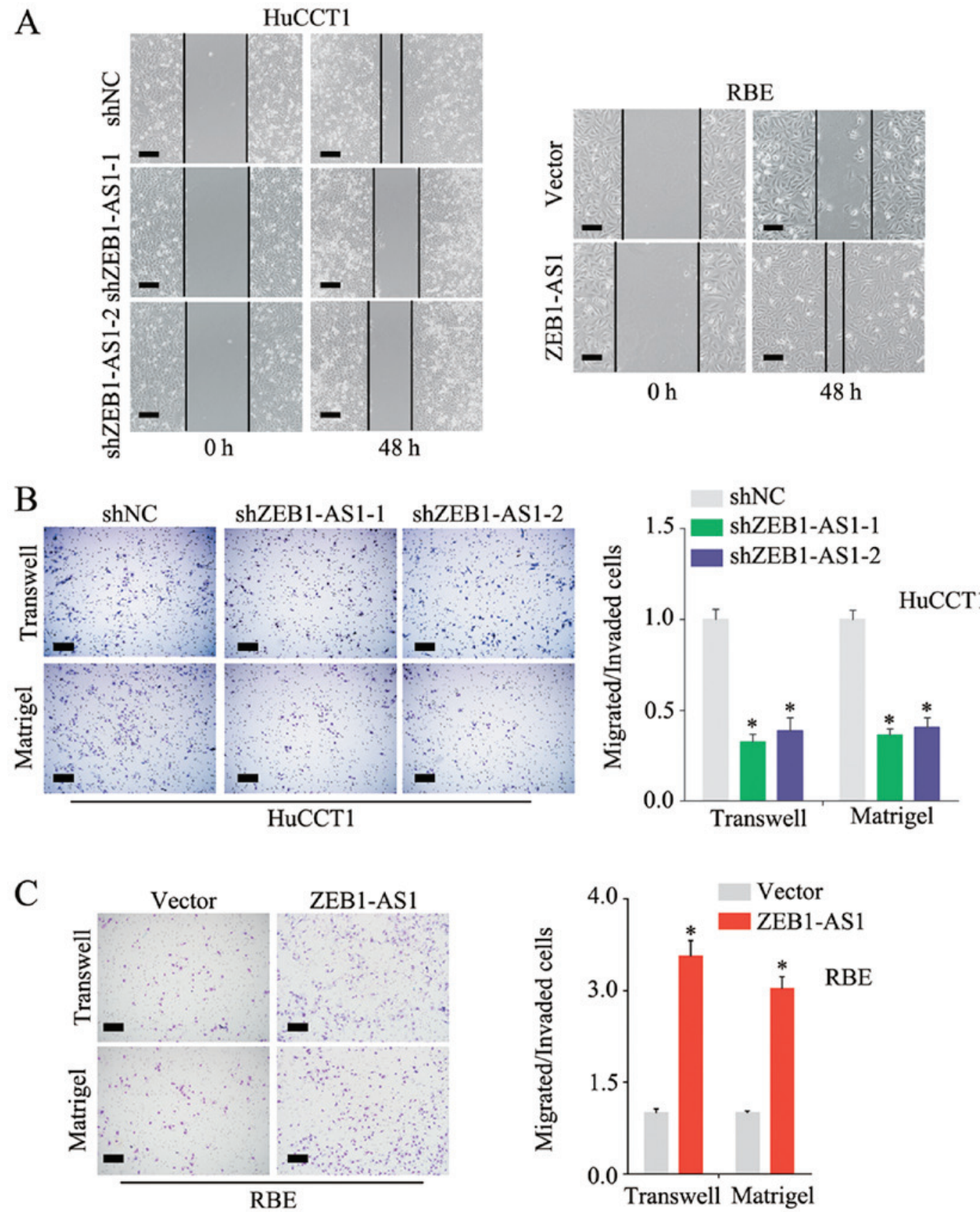

Figure 3. ZEB1-AS1 accelerates the migration and invasion of IHCC cells. (A) A wound healing assay was used to detect the migratory ability of HuCCT1 cells with ZEB1-AS1 knockdown and RBE cells with ZEB1-AS1 overexpression. (B) Migratory and invasive abilities were compared between the HuCCT1 cells with ZEB1-AS1 knockdown and control cells. (C) Migratory and invasive abilities were compared between RBE cells with ZEB1-AS1 overexpression and control cells. Scale bars, $500 \mu \mathrm{m}$ (A, B and C). "P<0.05. ZEB1-AS1, lncRNA ZEB1 antisense 1; IHCC, intrahepatic cholangiocarcinoma.

invasion $(\mathrm{P}=0.020)$, lymphatic metastasis $(\mathrm{P}=0.036)$ and an advanced TNM stage $(\mathrm{P}=0.037)$ (Table $\mathrm{I})$. These results suggest that the overexpression of ZEB1-AS1 indicates the clinical progression of IHCC.

To further elucidate the clinical significance of ZEB1-AS1 in IHCC, the prognosis of patients in the high and low ZEB1-AS1 expression groups was compared. Patients with a high ZEB1-AS1 expression had substantially lower OS and PFS rates than patients with a low ZEB1-AS1 expression (Fig. 1C and D). Additionally, the risk factors for poor OS and PFS were detected by univariate analysis and the identified 6 risk factors were subjected to multivariate analysis to discover independent risk factors for poor OS and PFS (Table II). A high ZEB1-AS1 expression was revealed to be an independent risk factor for poor OS (HR, 1.157; 95\% CI, 1.059-1.265; $\mathrm{P}=0.001)$ and PFS (HR, 1.131; 95\% CI, 1.038-1.232; $\mathrm{P}=0.005)$ (Table III). These results indicate that high ZEB1-AS1 expression is a potential biomarker for the prediction of a poor prognosis in IHCC.

ZEB1-AS1 accelerates the proliferation, migration and invasion of IHCC cells. As shown in Fig. 1A, among the 5 IHCC cell lines examined, the HuCCT1 line had the highest expression level of ZEB1-AS1, and the RBE line had the lowest. To better investigate the function of ZEB1-AS1 in IHCC, the knockdown and stable overexpression of ZEB1-AS1 was performed in the 
A

\begin{tabular}{lc}
\hline & $\begin{array}{c}\text { Number of mice with } \\
\text { distant metastasis }\end{array}$ \\
\hline shNC & $6 / 6$ \\
shZEB1-AS1-1 & $2 / 6$ \\
Vector & $1 / 6$ \\
ZEB1-AS1 & $5 / 6$ \\
\hline
\end{tabular}
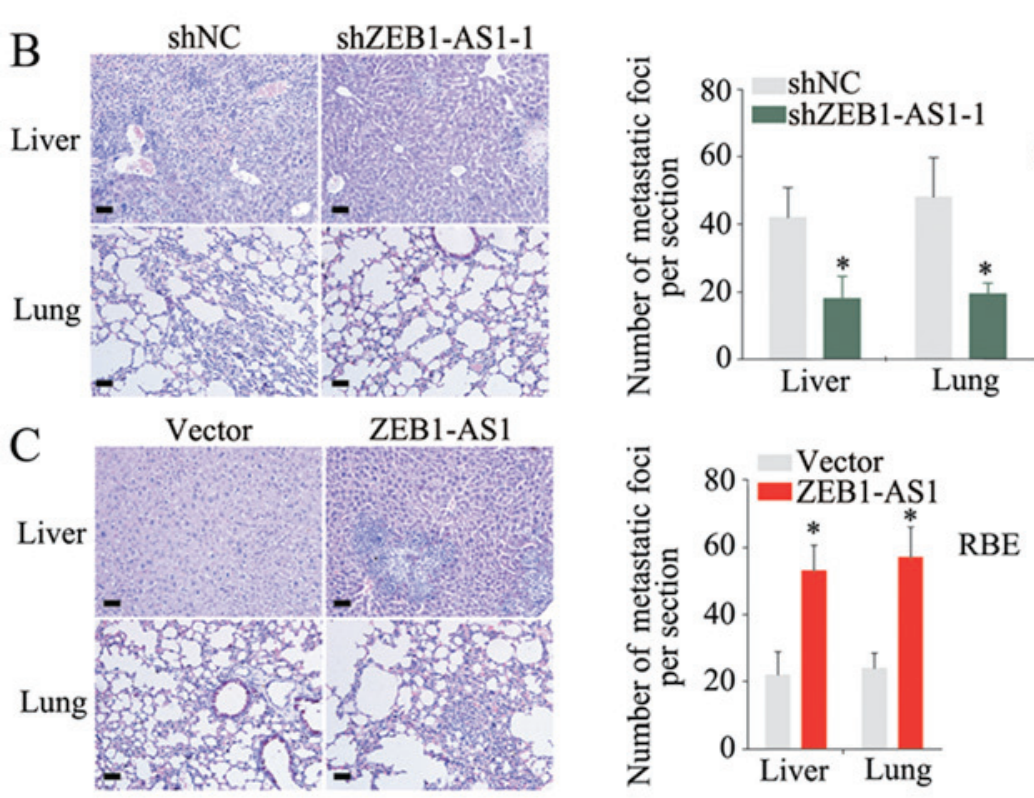

$\mathrm{D}$

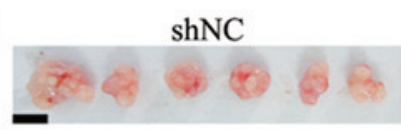

shZEB1-AS1-1
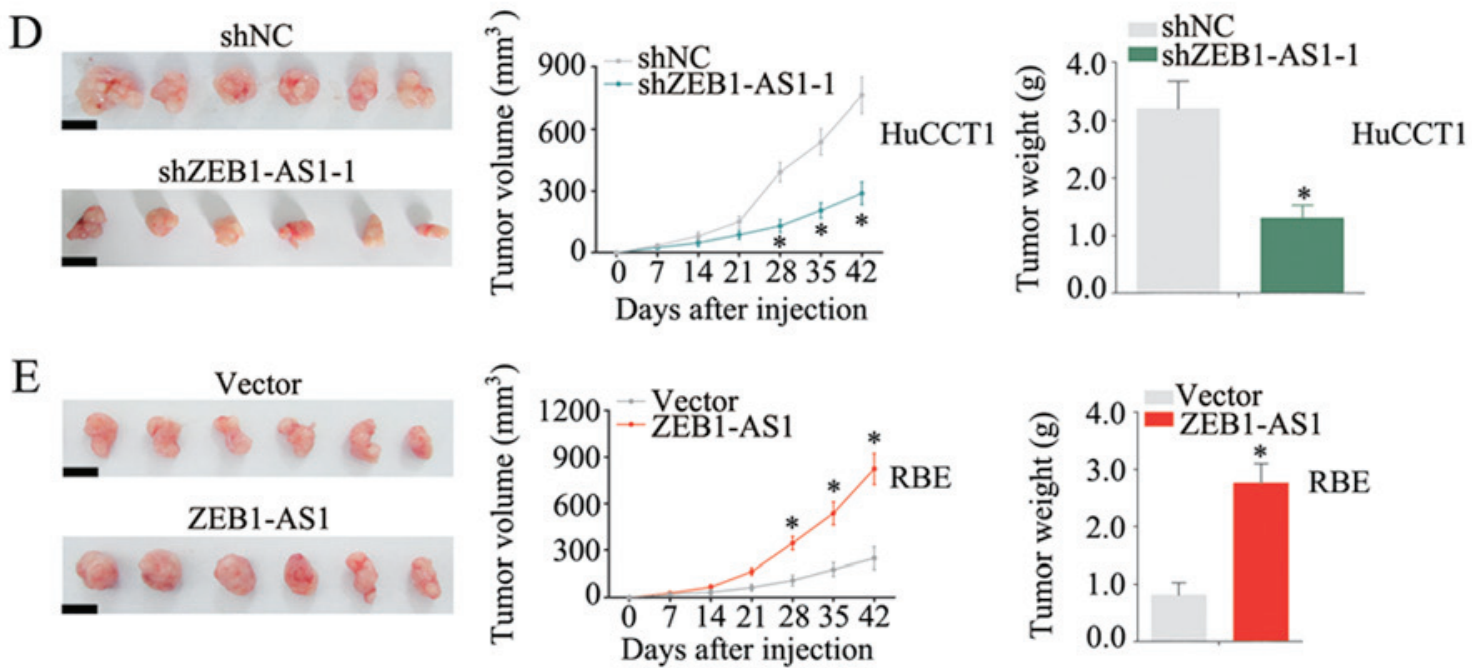

Figure 4. ZEB1-AS1 promotes tumor metastasis and growth in vivo. (A) Number of mice with distant metastasis. (B) Quantification of metastatic foci per section in the liver and lung of individual mice following the injection of HuCCT1 cells with ZEB1-AS1 knockdown or control cells. Representative images are shown. (C) Quantification of metastatic foci per section in the liver and lung of individual mice following the injection of RBE cells with ZEB1-AS1 overexpression or control cells. Representative images are shown. (D) Quantification of tumor volume and tumor weight of individual mice following the injection of HuCCT1 cells with ZEB1-AS1 knockdown or control cells. Representative images are shown. (E) Quantification of tumor volume and tumor weight of individual mice following the injection of RBE cells with ZEB1-AS1 overexpression or control cells. Representative images are shown. Scale bars, $20 \mu \mathrm{m}$ (B and C), and $1 \mathrm{~cm}$ (D and E). "P<0.05. ZEB1-AS1, lncRNA ZEB1 antisense 1; IHCC, intrahepatic cholangiocarcinoma.

HuCCT1 and RBE cells, respectively. (Fig. 2A) Notably, the HuCCT1 cells with ZEB1-AS1 deficiency exhibited a substantially reduced proliferative ability in the colony formation and CCK-8 assays (Fig. 2B and C). Consistently, the ectopic expression of ZEB1-AS1 significantly increased the proliferative ability of the RBE cells (Fig. 2D and E). In the wound healing assay, the knockdown of ZEB1-AS1 inhibited the migration of the HuCCT1 cells and the overexpression of ZEB1-AS1 promoted the migration of RBE cells (Fig. 3A); these results were confirmed by the Transwell assay (Fig. 3B and C, upper panels). Additional experiments demonstrated that ZEB1-AS1 accelerated the invasion of IHCC cells (Fig. 3B and C, lower panels) These findings demonstrate that ZEB1-AS1 promotes the proliferation, migration and invasion of IHCC cells. 
A

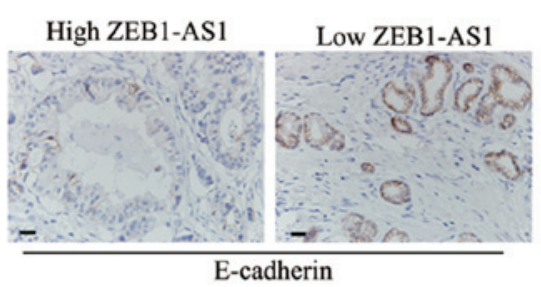

B

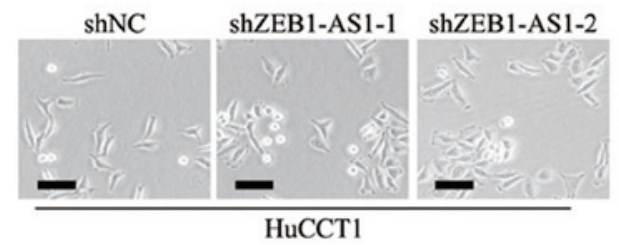

C

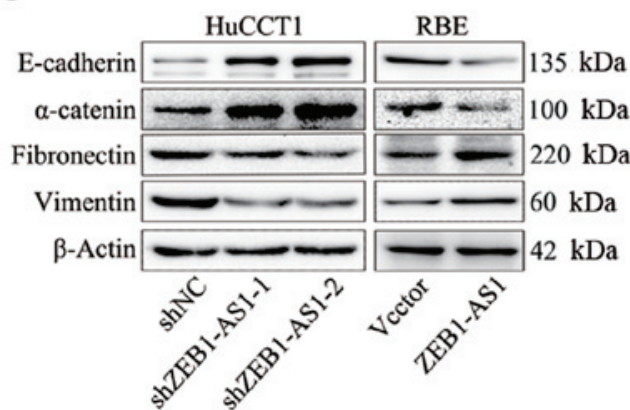

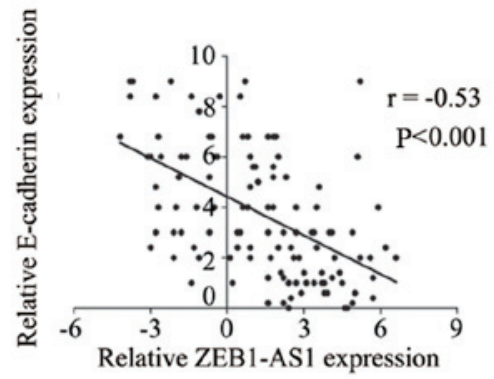

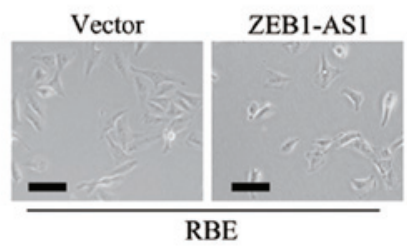

D

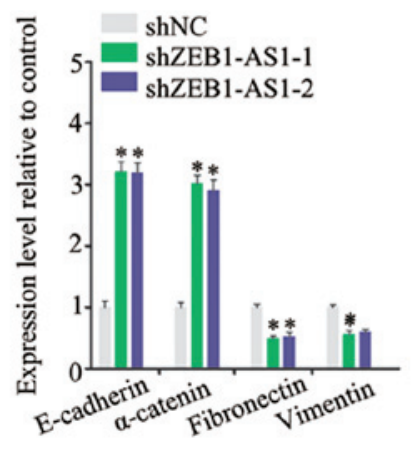

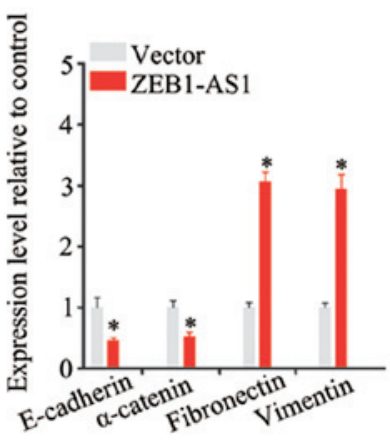

Figure 5.ZEB1-AS1 induces EMT in IHCC. (A) Spearman's correlation analysis of ZEB1-AS1 and E-cadherin expression in IHCC. Representative IHC images of E-cadherin are shown. (B) Phase-contrast microscopic images of HuCCT1 cells with ZEB1-AS1 knockdown, RBE cells with ZEB1-AS1 overexpression and control cells. (C) Protein levels of epithelial markers (E-cadherin and $\alpha$-catenin) and mesenchymal markers (fibronectin and vimentin) in HuCCT1 cells with ZEB1-AS1 knockdown, RBE cells with ZEB1-AS1 overexpression and control cells measured by western blot analysis. (D) RNA levels of epithelial markers (E-cadherin and $\alpha$-catenin) and mesenchymal markers (fibronectin and vimentin) in HuCCT1 cells with ZEB1-AS1 knockdown, RBE cells with ZEB1-AS1 overexpression and control cells detected by RT-qPCR. Scale bars, $25 \mu \mathrm{m}$ (A and B). * P<0.05. ZEB1-AS1, lncRNA ZEB1 antisense 1; IHCC, intrahepatic cholangiocarcinoma; EMT, epithelial-mesenchymal transition.

ZEB1-AS1 promotes IHCC metastasis and growth in vivo. To investigate the function of ZEB1-AS1, in vivo assays were performed. The knockdown of ZEB1-AS1 decreased the number of mice with distant metastasis, whereas the overexpression of ZEB1-AS1 increased this number (Fig. 4A). Moreover, the metastatic ability of the HuCCT1 cells was significantly inhibited when ZEB1-AS1 was knocked down (Fig. 4B), and ectopic expression of ZEB1-AS1 increased the number of metastatic foci in liver and lungs (Fig. 4C). Additionally, a decreased tumor size and weight were observed when ZEB1-AS1 was knocked down (Fig. 4D), whereas the overexpression of ZEB1-AS1 markedly increased the tumorigenicity of IHCC cells (Fig. 4E). These results suggest that ZEB1-AS1 promotes the metastasis and growth of IHCC.

ZEB1-AS1 induces EMT in IHCC. EMT is a fundamental process of cancer metastasis (23). In the present study, to explore the mechanisms underlying ZEB1-AS1 promotion of metastasis in IHCC, the role of ZEB1-AS1 in EMT was investigated. The expression of E-cadherin, a marker of EMT (30), was measured by IHC in IHCC tissues, and a negative association was found between the expression of ZEB1-AS1 and E-cadherin (Fig. 5A, left panel). In addition, Spearman's correlation analysis revealed that there was a negative correla- tion between ZEB1-AS1 and E-cadherin expression in IHCC (Fig. 5A, right panel). Moreover, the HuCCT1 cells in which ZEB1-AS1 was silenced displayed an epithelial morphology and formed colonies with increased intercellular contacts (Fig. 5B, left panels). Consistently, the overexpression of ZEB1-AS1 in the RBE cells induced a mesenchymal phenotype and reduced intercellular contacts compared with that in the control cells, which is a typical feature of cells undergoing EMT (Fig. 5B, right panels) Furthermore, the results of western blot analysis and RT-qPCR revealed that the expression of ZEB1-AS1 was positively associated with mesenchymal biomarkers (fibronectin, vimentin and N-cadherin) and negatively associated with epithelial biomarkers (E-cadherin and $\alpha$-catenin) (Fig. 5C and D). Based on these observations, it was concluded that ZEB1-AS1 induces EMT in IHCC.

ZEB1-AS1 regulates EMT through the miR-200al ZEB1 signaling pathway. To enhance our understanding of ZEB1-AS1, the subcellular localization of ZEB1-AS1 was detected via assays. The analysis of the nuclear and cytoplasmic ZEB1-AS1 RNA levels by RT-qPCR revealed that ZEB1-AS1 was located in the nucleus and cytoplasm in HuCCT1 and RBE cells (Fig. 6A). Competing with miRNAs is a primary mechanism of IncRNA functions (31). It has 


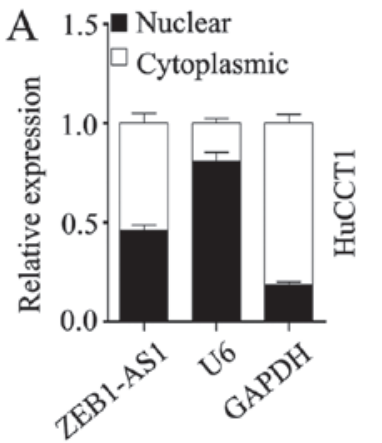

$\mathrm{C}$

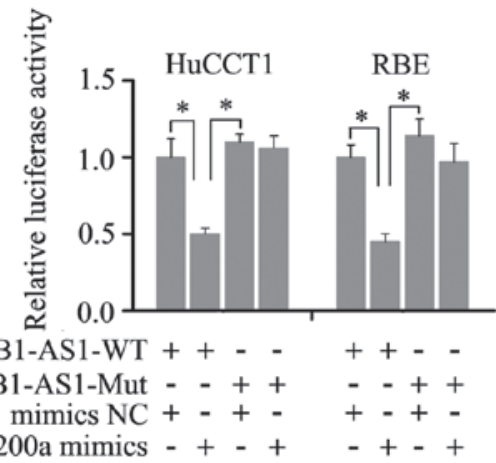

miR-200a mimics - + - + - + +

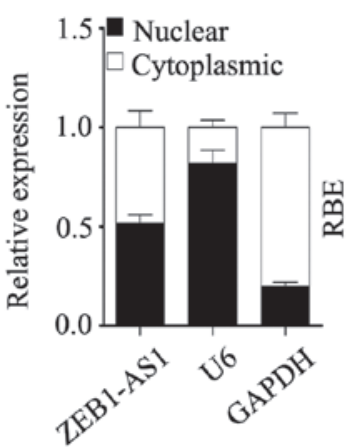

$\mathrm{B}$

$\mathrm{D}$
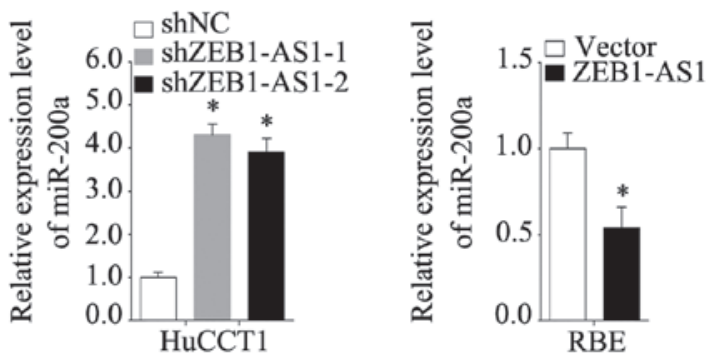

E

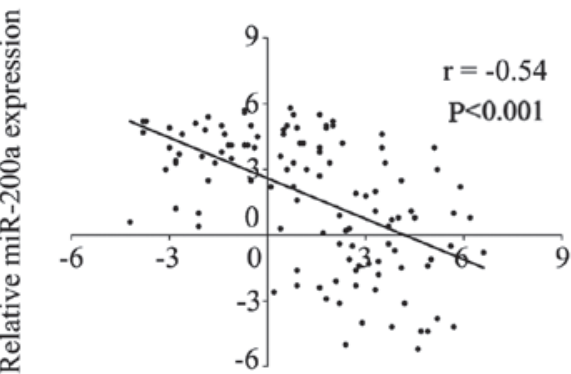

Relative ZEB1-AS1 expression

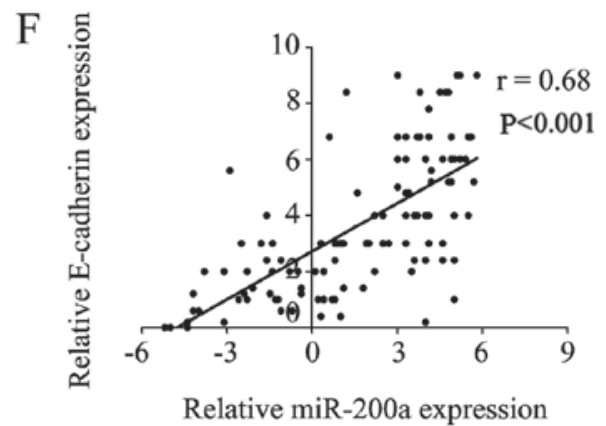

Figure 6. ZEB1-AS1 serves as a competing endogenous RNA and negatively regulates miR-200a expression in IHCC cells. (A) Subcellular localization of ZEB1-AS1 was detected by quantifying nuclear/cytoplasmic RNA fractions. (B) The potential binding site of ZEB1-AS1 and miR-200a predicted by a bioinformatics tool (starBase 2.0). (C) Dual-luciferase reporter assay was performed to detect the binding of miR-200a and ZEB1-AS1. (D) Relative expression levels of miR-200a in HuCCT1 cells with ZEB1-AS1 knockdown, RBE cells with ZEB1-AS1 overexpression and control cells. (E) Spearman's correlation analysis of ZEB1-AS1 and miR-200a in IHCC. (F) Spearman's correlation analysis of miR-200a and E-cadherin in IHCC. "P<0.05. ZEB1-AS1, lncRNA ZEB1 antisense 1; IHCC, intrahepatic cholangiocarcinoma.

previously been demonstrated that ZEB1-AS1 can sponge multiple miRNAs (32). The subcellular location of ZEB1-AS1 indicates that ZEB1-AS1 may function by competing with miRNAs. Thus, in the present study, potential miRNA targets of ZEB1-AS1 were predicted using the bioinformatics tool starBase, and miR-200a was predicted to be a potential target of ZEB1-AS1 (Fig. 6B). It was found that miR-200a mimics markedly reduced the luciferase activity of ZEB1-AS1-WT, but had no effect on ZEB1-AS1-Mut (Fig. 6C). Moreover, miR-200a expression was increased in the HuCCT1 cells in which ZEB1-AS1 was knocked down and decreased in the RBE cells in which ZEB1-AS1 was overexpressed (Fig. 6D). Additionally, the level of miR-200a negatively correlated with ZEB1-AS1 in the IHCC tissues (Fig. 6E). Furthermore, a strong positive correlation was found to exist between miR-200a and E-cadherin expression in IHCC tissues (Fig. 6F), which indicates that miR-200a may influence EMT in IHCC. As expected, transfection with miR-200a mimics reversed the downregula- tion of E-cadherin and the upregulation of vimentin induced by the overexpression of ZEB1-AS1, as evidenced by the results of western blot analysis and RT-qPCR (Fig. 7A and B). Consistently, transfection with miR-200a inhibitor blocked the increased expression of E-cadherin and the decreased expression of vimentin in the HuCCT1 cells in which ZEB1-AS1 was knocked down (Fig. 7A and B). These results demonstrate that miR-200a blocks the EMT induced by ZEB1-AS1.

ZEB1, a master regulator of EMT (26), is a potential target of miR-200a $(33,34)$. Previous studies have reported that ZEB1-AS1 can function through ZEB1 $(32,35,36)$. Thus, the present study evaluated the expression of ZEB1 by IHC; the results revealed indicated a negative association between miR-200a and ZEB1 (Fig. 7C, left panels). In addition, Spearman's correlation analysis also revealed a negative correlation between ZEB1 and miR-200a expression (Fig. 7C, right panel). Transfection with miR-200a mimics reversed the upregulated expression of ZEB1 induced by ZEB1-AS1 
A
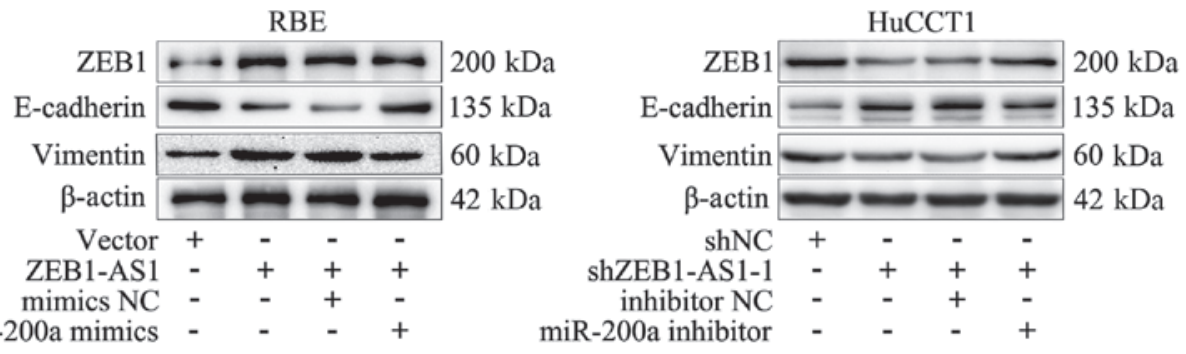

$\mathrm{B}$
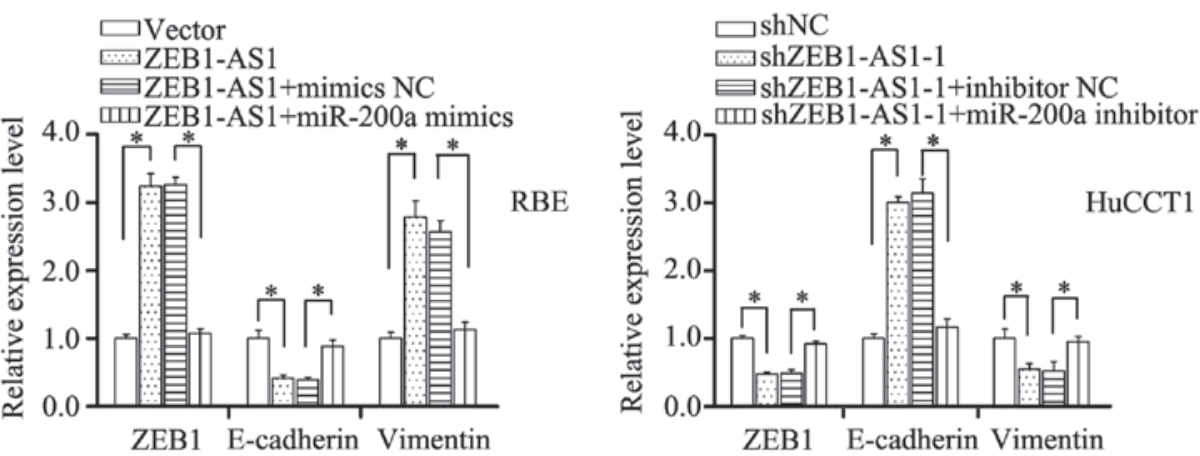

$\mathrm{C}$
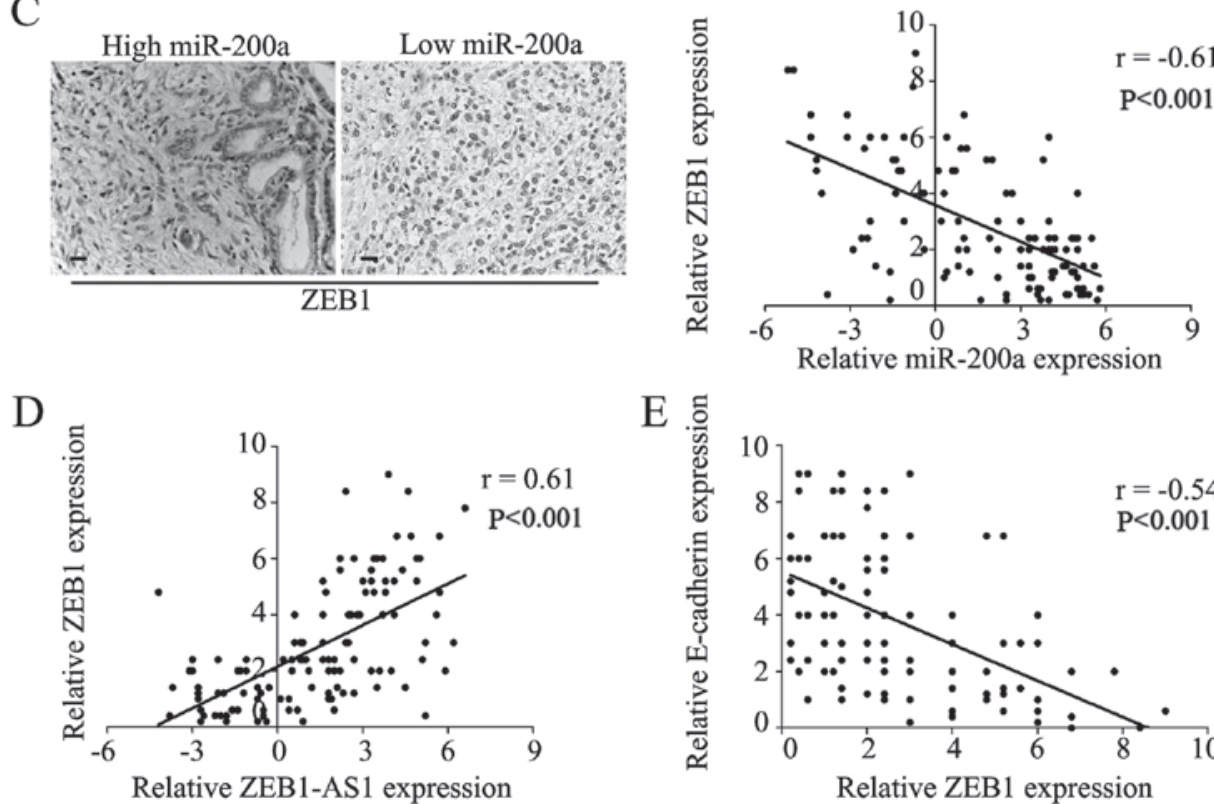

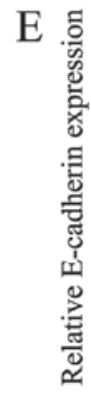

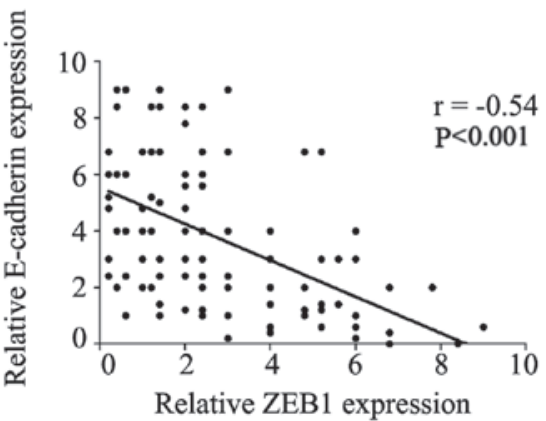

Figure 7.ZEB1-AS1 induces EMT through miR-200a/ZEB1 signaling in IHCC. (A) To detect the effect of miR-200a on EMT induction by ZEB1-AS1, western blot analysis was performed to detect the protein levels of ZEB1, E-cadherin and vimentin in RBE cells and HuCCT1 cells. (B) To detect the effect of miR-200a on EMT induction by ZEB1-AS1, RT-qPCR was used to detect the RNA levels of ZEB1, E-cadherin and vimentin in RBE cells and HuCCT1 cells. (C) Linear regression analysis of miR-200a and ZEB1 in IHCC. Representative IHCC images of ZEB1 are shown. (D) Spearman's correlation analysis of ZEB1-AS1 and ZEB1 in IHCC. (E) Spearman's correlation analysis of ZEB1 and E-cadherin in IHCC. Scale bars, $25 \mu \mathrm{m}$ (A and B). "P<0.05. ZEB1-AS1, lncRNA ZEB1 antisense 1; IHCC, intrahepatic cholangiocarcinoma; EMT, epithelial-mesenchymal transition.

overexpression, and transfection with miR-200a inhibitor restored the reduced expression of ZEB1 induced by ZEB1-AS1 silencing (Fig. 7A and B). Moreover, the expression of ZEB1 positively correlated with that of ZEB1-AS1, but negatively correlated with E-cadherin expression in the IHCC tissues (Fig. 7D and E). These findings demonstrate that ZEB1-AS1 induces EMT through the miR-200a/ZEB1 signaling pathway.

\section{Discussion}

The findings of the present study demonstrated that ZEB1-AS1 was overexpressed in IHCC and promoted IHCC proliferation and metastasis in vitro and in vivo. Moreover, a high ZEB1-AS1 expression was associated with microvascular invasion, lymphatic metastasis and an advanced TNM stage; all these factors are pivotal in influencing the survival of patients with IHCC. The log-rank test revealed that patients with a high ZEB1-AS1 expression had considerably lower OS and PFS rates than patients with a low ZEB1-AS1 expression. Additionally, a high ZEB1-AS1 expression was identified as an independent risk factor for low OS and PFS. A previous meta-analysis aimed at discovering the prognostic value of ZEB1-AS1 in cancer, which included 11 studies representing 891 cancer patients, indicated that a high ZEB1-AS1 
expression was an unfavorable predictor of cancer prognosis in terms of OS, disease-free survival and recurrence-free survival (37). Taken together, these findings indicate that ZEB1-AS1 functions as an oncogene in cancer and may be a promising prognostic biomarker for cancer patients including patients with IHCC.

In cholangiocarcinoma, EMT plays a prominent role in cancer progression and may lead to highly metastatic and desmoplastic stroma (30). EMT biomarkers have prognostic values in cholangiocarcinoma (30). Additionally, EMT pathway-targeted therapy is regarded as a potential IHCC treatment $(38,39)$. The present study revealed that the level of ZEB1-AS1 expression was associated with morphological changes in IHCC cells. Moreover, the level of ZEB1-AS1 was found to be positively associated with epithelial biomarkers and negatively associated with mesenchymal biomarkers at the protein and mRNA level, suggesting that ZEB1-AS1 may regulate EMT at the transcriptional level. Further experiments were performed to confirm this hypothesis. As presented in the current study, no statistically significant differences were observed between the expression of ZEB1-AS1 and the number of tumors and tumor size in the clinical specimens; however, ZEB-AS1 accelerated cell proliferation in colony formation and CCK-8 assays. This may be caused by the differences between the in vitro assays and the human body.

miR-200a is a member of the miR-200 family (40). Several studies have demonstrated that miR-200 family members are downregulated during tumor progression and act as key inhibitors of EMT $(25,26,41,42)$. In addition, they can target the transcriptional factors of EMT (ZEB1 and ZEB2) and thereby modulate the expression of E-cadherin $(33,34)$. In cholangiocarcinoma, $\mathrm{miR}-200 \mathrm{~b} / \mathrm{c}$ has been reported to mediate the migration and invasion by directly targeting rho-kinase 2 (43). Moreover, DLC1 has been demonstrated to be a potential target of miR-200a and miR-429 may target FBXW7 and CDH6 in cholangiocarcinoma (44). Additionally, miR-200a has been shown to suppress the invasive ability of cholangiocarcinoma cells (45). However, the mechanisms underlying the regulatory functions of miR-200 family members functions in IHCC require further investigation. The present study found that ZEB1-AS1 mediated the expression of ZEB1 by competing with miR-200a; this finding was confirmed by luciferase reporter assay. Moreover, a negative correlation was observed between ZEB1-AS1 and miR-200a expression in IHCC cells and tissues. Furthermore, miR-200a reversed the effects of an enhanced or reduced ZEB1-AS1 expression. These results confirm the role of miR-200a in mediating the function of ZEB1-AS1.

In conclusion, the findings of the present study demonstrate that the upregulation of ZEB1-AS1 promotes IHCC progression by accelerating proliferation and metastasis. Moreover, high ZEB1-AS1 expression indicates poor OS and PFS rates, and serves as an independent risk factor for poor OS and PFS. ZEB1-AS1 acts as molecular sponge for miR-200a and regulates the expression of its target gene ZEB1. These findings contribute to the better understanding of the mechanisms underlying IHCC progression. ZEBA-AS1 may be a promising biomarker for the prediction of prognosis and a potential target for IHCC treatment.

\section{Acknowledgements}

Not applicable.

\section{Funding}

The present study was supported by the National Natural Sciences Foundation of China (grant nos. 81903044 and 31701013), and the Natural Sciences Foundation of Shandong Province (grant no. ZR2017BC032).

\section{Availability of data and materials}

The datasets used and/or analyzed during the current study are available from the corresponding author on reasonable request.

\section{Authors' contributions}

MiJ, SN, JC and HY were involved in the conceptualization of the study. ZC was involved in data curation. SN and HY were responsible for funding acquisition. JC, LC, MeJ, LG and WM were involved in the study methodology. MiJ, SN, $\mathrm{JC}$ and LG were involved in project administration. ZC was responsible for obtaining resources. MiJ, LC and WM were involved in data validation. MeJ was invovled in the acquisition of the software used. HY was involved in the writing of the original draft, and in the writing, reviewing and editing of the manuscript. All authors have read and approved the final manuscript.

\section{Ethics approval and consent to participate}

The research protocol conformed to the principles outlined in the Declaration of Helsinki. Each patient provided written and signed informed consent and the protocol of the study was approved by the Ethics Committee of Shandong Provincial Qianfoshan Hospital, the First Hospital Affiliated with Shandong First Medical University. The animal experiment was approved by the Ethics Committee of Shandong Provincial Qianfoshan Hospital.

\section{Patient consent for publication}

Not applicable.

\section{Competing interests}

The authors declare that they have no competing interests.

\section{References}

1. Sirica AE, Gores GJ, Groopman JD, Selaru FM, Strazzabosco M, Wei Wang $X$ and Zhu AX: Intrahepatic cholangiocarcinoma: Continuing challenges and translational advances. Hepatology 69: 1803-1815, 2019.

2. Squires MH, Cloyd JM, Dillhoff M, Schmidt C and Pawlik TM: Challenges of surgical management of intrahepatic cholangiocarcinoma. Expert Rev Gastroenterol Hepatol 12: 671-681, 2018.

3. Mavros MN, Economopoulos KP, Alexiou VG and Pawlik TM: Treatment and prognosis for patients with intrahepatic cholangiocarcinoma: Systematic review and meta-analysis. JAMA Surg 149: 565-574, 2014.

4. Shaib Y and El-Serag HB: The epidemiology of cholangiocarcinoma. Semin Liver Dis 24: 115-125, 2004. 
5. Farges O, Fuks D, Boleslawski E, Le Treut YP, Castaing D, Laurent A, Ducerf C, Rivoire M, Bachellier P, Chiche L, et al: Influence of surgical margins on outcome in patients with intrahepatic cholangiocarcinoma: A multicenter study by the AFC-IHCC-2009 study group. Ann Surg 254: 824-830, 2011

6. de Jong MC, Nathan H, Sotiropoulos GC, Paul A, Alexandrescu S, Marques H, Pulitano C, Barroso E, Clary BM Aldrighetti L, et al: Intrahepatic cholangiocarcinoma: An international multi-institutional analysis of prognostic factors and lymph node assessment. J Clin Oncol 29: 3140-3145, 2011.

7. Moeini A, Sia D, Bardeesy N, Mazzaferro V and Llovet JM Molecular pathogenesis and targeted therapies for intrahepatic cholangiocarcinoma. Clin Cancer Res 22: 291-300, 2016.

8. Gutschner T and Diederichs S: The hallmarks of cancer: A long non-coding RNA point of view. RNA Biol 9: 703-719, 2012.

9. Ulitsky I and Bartel DP: LincRNAs: Genomics, evolution, and mechanisms. Cell 154: 26-46, 2013.

10. Tang Y, Cheung BB, Atmadibrata B, Marshall GM, Dinger ME, Liu PY and Liu T: The regulatory role of long noncoding RNAs in cancer. Cancer Lett 391: 12-19, 2017

11. Gutschner T, Hammerle M and Diederichs S: MALAT1-a paradigm for long noncoding RNA function in cancer. J Mol Med (Berl) 91: 791-801, 2013.

12. Zou Y,Zhong Y, Wu J, Xiao H, Zhang X, Liao X, Li J, Mao X, Liu Y and Zhang F: Long non-coding PANDAR as a novel biomarker in human cancer: A systematic review. Cell Prolif 51: e12422, 2018.

13. Chen JS, Wang YF, Zhang XQ, Lv JM, Li Y, Liu XX and Xu TP: H19 serves as a diagnostic biomarker and up-regulation of H19 expression contributes to poor prognosis in patients with gastric cancer. Neoplasma 63: 223-230, 2016.

14. Zhang L, Yang Z, Trottier J, Barbier O and Wang L: Long noncoding RNA MEG3 induces cholestatic liver injury by interaction with PTBP1 to facilitate shp mRNA decay. Hepatology 65 : 604-615, 2017.

15. Li T, Xie J, Shen C, Cheng D, Shi Y, Wu Z, Deng X, Chen H, Shen B, Peng C, et al: Upregulation of long noncoding RNA ZEB1-AS1 promotes tumor metastasis and predicts poor prognosis in hepatocellular carcinoma. Oncogene 35: 1575-1584, 2016.

16. Lv QL, Hu L, Chen SH, Sun B, Fu ML, Qin CZ, Qu Q, Wang GH, $\mathrm{He} \mathrm{CJ}$ and Zhou HH: A long noncoding RNA ZEB1-AS promotes tumorigenesis and predicts poor prognosis in glioma. Int J Mol Sci 17: pii: E1431, 2016.

17. Fu J and Cui Y: Long noncoding RNA ZEB1-AS1 expression predicts progression and poor prognosis of colorectal cancer. Int J Biol Markers 32: e428-e433, 2017

18. Li Y, Wen X, Wang L, Sun X, Ma H, Fu Z and Li L: LncRNA ZEB1-AS1 predicts unfavorable prognosis in gastric cancer. Surg Oncol 26: 527-534, 2017.

19. Su W, Xu M, Chen X, Chen N, Gong J, Nie L, Li L, Li X, Zhang M and Zhou Q: Long noncoding RNA ZEB1-AS1 epigenetically regulates the expressions of ZEB1 and downstream molecules in prostate cancer. Mol Cancer 16: 142, 2017.

20. Cheng R, Li N, Yang S, Liu L and Han S: Long non-coding RNA ZEB1-AS1 promotes cell invasion and epithelial to mesenchymal transition through inducing ZEB1 expression in cervical cancer. Onco Targets Ther 11: 7245-7253, 2018.

21. Ni Y, Fang J, Zhu L, Jiang H, Liu Y, Miao R, Shao C and Shao S: The significant prognostic value of ZEB1-AS1 up-regulation in patients with cancer. J Cancer 9: 2502-2509, 2018.

22. Wu Y, Ding M, Wei S, Wu T, Xu R, Zhu X and Liu H: The prognostic value of long noncoding RNA ZEB1-AS1 on clinical outcomes in human cancer. J Cancer 9: 3690-3698, 2018.

23. Pastushenko I and Blanpain C: EMT transition states during tumor progression and metastasis. Trends Cell Biol 29: 212-226, 2019.

24. Dongre A and Weinberg RA: New insights into the mechanisms of epithelial-mesenchymal transition and implications for cancer. Nat Rev Mol Cell Biol 20: 69-84, 2019.

25. Brabletz S and Brabletz T: The ZEB/miR-200 feedback loop-a motor of cellular plasticity in development and cancer? EMBO Rep 11: 670-677, 2010.

26. Hill L, Browne G and Tulchinsky E: ZEB/miR-200 feedback loop: At the crossroads of signal transduction in cancer. Int J Cancer 132: 745-754, 2013
27. Ueno M, Morizane C, Ikeda M, Okusaka T, Ishii H and Furuse J: A review of changes to and clinical implications of the eighth TNM classification of hepatobiliary and pancreatic cancers. Jpn J Clin Oncol 49: 1073-1082, 2019.

28. Yang H, Lu X, Liu Z, Chen L, Xu Y, Wang Y, Wei G and Chen Y: FBXW7 suppresses epithelial-mesenchymal transition, stemness and metastatic potential of cholangiocarcinoma cells. Oncotarget 6: 6310-6325, 2015.

29. Livak KJ and Schmittgen TD: Analysis of relative gene expression data using real-time quantitative PCR and the 2(-Delta Delta $\mathrm{C}(\mathrm{T})$ ) method. Methods 25: 402-408, 2001.

30. Vaquero J, Guedj N, Claperon A, Nguyen Ho-Bouldoires TH, Paradis V and Fouassier L: Epithelial-mesenchymal transition in cholangiocarcinoma: From clinical evidence to regulatory networks. J Hepatol 66: 424-441, 2017.

31. Bartonicek N, Maag JL and Dinger ME: Long noncoding RNAs in cancer: Mechanisms of action and technological advancements. Mol Cancer 15: 43, 2016.

32. Li J, Li Z, Leng K, Xu Y, Ji D, Huang L, Cui Y and Jiang X: ZEB1-AS1: A crucial cancer-related long non-coding RNA. Cell Prolif 51: e12423, 2018.

33. Park SM, Gaur AB, Lengyel E and Peter ME: The miR-200 family determines the epithelial phenotype of cancer cells by targeting the E-cadherin repressors ZEB1 and ZEB2. Genes Dev 22: 894-907, 2008.

34. Gregory PA, Bert AG, Paterson EL, Barry SC, Tsykin A, Farshid G, Vadas MA, Khew-Goodall Y and Goodall GJ: The miR-200 family and miR-205 regulate epithelial to mesenchymal transition by targeting ZEB1 and SIP1. Nat Cell Biol 10: 593-601, 2008.

35. Qian W, Cai X, Qian Q, Peng W, Yu J, Zhang X, Tian L and Wang C: LncRNA ZEB1-AS1 promotes pulmonary fibrosis through ZEB1-mediated epithelial-mesenchymal transition by competitively binding miR-141-3p. Cell Death Dis 10: 129, 2019

36. QuR,ChenXandZhangC:LncRNAZEB1-AS1/miR-409-3p/ZEB1 feedback loop is involved in the progression of non-small cell lung cancer. Biochem Biophys Res Commun 507: 450-456, 2018.

37. Chen $\mathrm{C}$, Feng $\mathrm{Y}$ and Wang X: LncRNA ZEB1-AS1 expression in cancer prognosis: Review and meta-analysis. Clin Chim Acta 484: 265-271, 2018.

38. Hirose A, Tajima H, Ohta T, Tsukada T, Okamoto K, Nakanuma S, Sakai S, Kinoshita J, Makino I, Furukawa H, et al: Low-dose paclitaxel inhibits the induction of epidermal-mesenchymal transition in the human cholangiocarcinoma CCKS-1 cell line. Oncol Lett 6: 915-920, 2013.

39. Lu Z, Wang J, Zheng T, Liang Y, Yin D, Song R, Pei T, Pan S, Jiang H and Liu L: FTY720 inhibits proliferation and epithelial-mesenchymal transition in cholangiocarcinoma by inactivating STAT3 signaling. BMC Cancer 14: 783, 2014.

40. Senfter D, Madlener S, Krupitza G and Mader RM: The microRNA-200 family: Still much to discover. Biomol Concepts 7: 311-319, 2016.

41. Cano A and Nieto MA: Non-coding RNAs take centre stage in epithelial-to-mesenchymal transition. Trends Cell Biol 18: 357-359, 2008.

42. Gregory PA, Bracken CP, Bert AG and Goodall GJ: MicroRNAs as regulators of epithelial-mesenchymal transition. Cell Cycle 7: 3112-3118, 2008.

43. Peng F, Jiang J, Yu Y, Tian R, Guo X, Li X, Shen M, Xu M, Zhu F, Shi C, et al: Direct targeting of SUZ12/ROCK2 by miR-200b/c inhibits cholangiocarcinoma tumourigenesis and metastasis. Br J Cancer 109: 3092-3104, 2013

44. Goeppert B, Ernst C, Baer C, Roessler S, Renner M, Mehrabi A, Hafezi M, Pathil A, Warth A, Stenzinger A, et al: Cadherin-6 is a putative tumor suppressor and target of epigenetically dysregulated miR-429 in cholangiocarcinoma. Epigenetics 11: 780-790, 2016.

45. Chen C, Yang D, Wang Q and Wang X: Expression and clinical pathological significance of miR-200a in concurrent cholangiocarcinoma associated with hepatolithiasis. Med Sci Monit 21: 3585-3590, 2015.

This work is licensed under a Creative Commons Attribution-NonCommercial-NoDerivatives 4.0 International (CC BY-NC-ND 4.0) License. 